\title{
Recent results from the SND experiment at VEPP-2000 collider
}

\author{
A. S. Kupich ${ }^{\star a b}$ M. N. Achasov ${ }^{a b}$ A. Yu. Barnyakov ${ }^{a b}$ K. I. Beloborodov ${ }^{a b}$ \\ A. V. Berdyugin ${ }^{a b}$ A. G. Bogdanchikov ${ }^{a}$ A. A. Botov ${ }^{a}$ T. V. Dimova ${ }^{a b}$ V. P. Druzhinin ${ }^{a b}$ \\ V. B. Golubev ${ }^{a b}$ L. V. Kardapoltsev ${ }^{a b}$ A. G. Kharlamov ${ }^{a b}$ A. A. Korol ${ }^{a b}$ S. V. Koshuba ${ }^{a}$ \\ D. P. Kovrizhin ${ }^{a b}$ K. A. Martin ${ }^{a b}$ A. E. Obrazovsky ${ }^{a}$ E. V. Pakhtusova ${ }^{a}$ \\ S. I. Serednyakov ${ }^{a b}$ D. A. Shtol ${ }^{a b}$ Z. K. Silagadze ${ }^{a b}$ I. K. Surin ${ }^{a b}$ Yu. V. Usov ${ }^{a b}$ \\ A. V. Vasiljev ${ }^{a b}$ and the SND Collaboration \\ ${ }^{a}$ Budker Institute of Nuclear Physics, Novosibirsk, 630090, Russia \\ ${ }^{b}$ Novosibirsk State University, Novosibirsk, 630090, Russia \\ E-mail: a.s.kupicheinp.nsk.su
}

\begin{abstract}
Latest results on study of processes of $e^{+} e$ annihilation into exclusive hadronic states at $\sqrt{s}<2$ $\mathrm{GeV}$ obtained in the SND experiment at the VEPP-2M and VEPP-2000 colliders are presented. In particular, we announce results of the precise measurements of the $e^{+} e \rightarrow \pi^{0} \gamma$ and $e^{+} e \rightarrow K^{+} K^{-}$ cross sections, the first measurements of the $e^{+} e \rightarrow \omega \pi^{0} \eta$ and $e^{+} e \rightarrow \pi^{+} \pi^{-} \pi^{0} \eta$ reactions and preliminary results of measurement of the $e^{+} e \rightarrow \pi^{+} \pi^{-}$cross section in energy range $0.5<\sqrt{s}<$ $0.9 \mathrm{GeV}$.
\end{abstract}

XVII International Conference on Hadron Spectroscopy and Structure - Hadron2017

25-29 September, 2017

University of Salamanca, Salamanca, Spain

${ }^{*}$ Speaker. 


\section{Detector and experiment}

The SND $[1,2,3,4]$ is the general purpose nonmagnetic detector. Its main part is a spherical three-layer electromagnetic calorimeter containing $1640 \mathrm{NaI}(\mathrm{Tl})$ crystals. Directions of charged particles are measured by a tracking system based on a nine-layer drift chamber. The particle identification is provided by $d E / d x$ measurements in the tracking system and a system of aerogel Cherenkov counters. Outside the calorimeter a muon detector is located.

SND collected data at the VEPP-2M [5] and VEPP2000 [6] $e^{+} e^{-}$colliders. At VEPP-2M, data with an integrated luminosity of about $30 \mathrm{pb}^{-1}$ were recorded in 1996-2000 in the energy range 0.4-1.4 GeV. At VEPP2000, a wider energy interval, $0.3-2.0 \mathrm{GeV}$, is studied. A $69 \mathrm{pb}^{-1}$ data sample was collected during 2010-2013. Experiments with increased luminosity started in the end of 2016 after VEPP-2000 accelerator complex upgrade. About $50 \mathrm{pb}^{-1}$ of integrated luminosity has been already collected during the 2017 run.

The main goal of the SND experiments is careful measurement of exclusive hadronic cross sections below $2 \mathrm{GeV}$, which are, in particular, required for the Standard Model calculation of the muon $(g-2)$ and running $\alpha_{Q E D}$. Here the new results on measurement of five exclusive cross sections are presented.

\section{Precise cross-section measurements}

The $e^{+} e^{-} \rightarrow \pi^{0} \gamma$ cross section is the third largest cross section (after $e^{+} e^{-} \rightarrow \pi^{+} \pi^{-}$and $\pi^{+} \pi^{-} \pi^{0}$ ) below $1 \mathrm{GeV}$. Analysis of the $e^{+} e^{-} \rightarrow \pi^{0} \gamma$ data allows to extract the widths of vectormeson radiative decays, which are widely used in phenomenological models. Experiments at the VEPP-2M $e^{+} e^{-}$collider with the SND [7, 8] and CMD-2 [9] detectors have provided the most accurate data on this process. Previous SND results [7, 8] are based on about 25\% of VEPP-2M data. Here we present a new analysis [10] using the full SND@VEPP-2M data sample.

The measured $e^{+} e^{-} \rightarrow \pi^{0} \gamma$ cross section shown in Fig. 1 agrees with previous SND [7, 8] and CMD-2 [9] measurements within the systematic uncertainties. The fit to cross section provides the products of branching fractions:

$$
\begin{aligned}
B\left(\rho \rightarrow \pi^{0} \gamma\right) B\left(\rho \rightarrow e^{+} e^{-}\right) & =(1.98 \pm 0.22 \pm 0.10) \times 10^{-8} \\
B\left(\omega \rightarrow \pi^{0} \gamma\right) B\left(\omega \rightarrow e^{+} e^{-}\right) & =(6.336 \pm 0.056 \pm 0.089) \times 10^{-6}, \\
B\left(\phi \rightarrow \pi^{0} \gamma\right) B\left(\phi \rightarrow e^{+} e^{-}\right) & =(4.04 \pm 0.09 \pm 0.19) \times 10^{-7}
\end{aligned}
$$

For the $\omega$ meson, accuracy of the product is improved by a factor of 1.6. There was a tension between the value of the ratio $B\left(\omega \rightarrow \pi^{0} \gamma\right) / B\left(\omega \rightarrow \pi^{+} \pi^{-} \pi^{0}\right)$ measured by KLOE [11] and the same value obtained using VEPP-2M data. After our $\pi^{0} \gamma$ measurement this tension increases up to

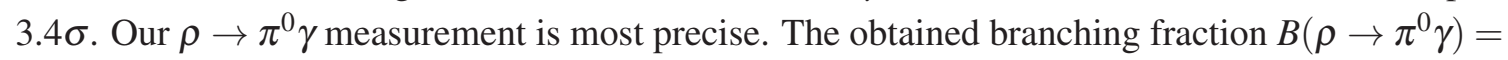
$(4.20 \pm 0.52) \times 10^{-4}$ is lower than the PDG value by $1.8 \sigma$, but agrees with the branching fraction for the charged $\rho$ decay. Our result for the $\phi \rightarrow \pi^{0} \gamma$ has an accuracy comparable with current PDG value.

Accuracy of $e^{+} e^{-} \rightarrow K^{+} K^{-}$cross section measurement in the energy range 1.05-2.00 GeV [12] was improved as well. SND result in comparison with most precise previous measurement by BABAR [13] is shown in Fig. 2 

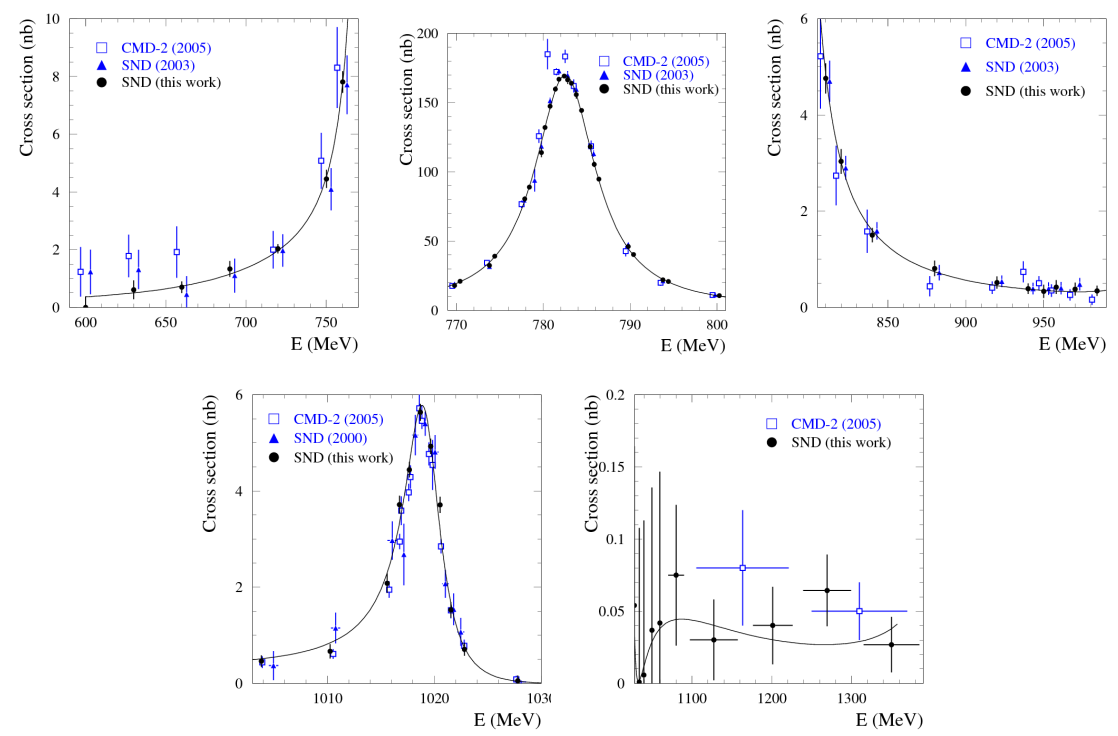

Figure 1: The $e^{+} e^{-} \rightarrow \pi^{0} \gamma$ cross section measured by SND using the full VEPP-2M data sample in comparison with the previous most accurate measurements. The curve is the result of the VMD fit. Only statistical errors are shown. The systematic errors are 3.2\%, 3\%, and 6\% for SND (2000), SND (2003), and CMD-2 (2005) data, respectively. Our systematic uncertainty at the $\omega$ and $\phi$ peaks is $1.4 \%$.
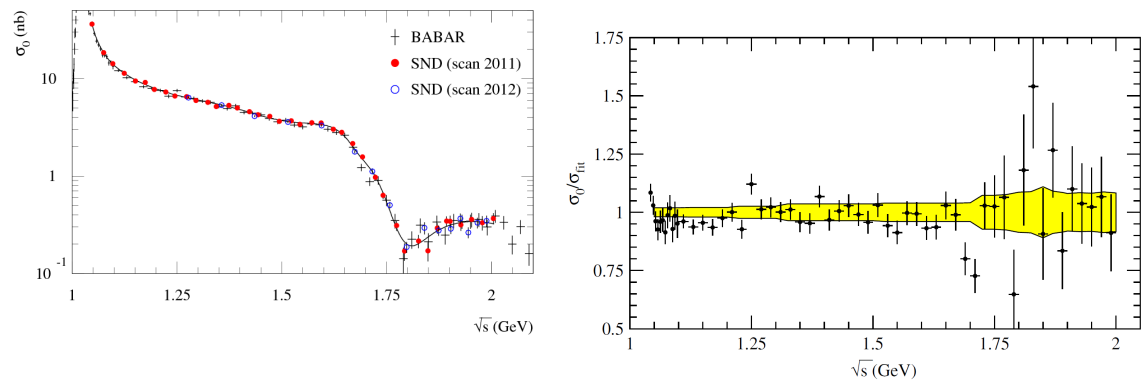

Figure 2: Left panel: The $e^{+} e^{-} \rightarrow K^{+} K^{-}$cross section measured by SND at VEPP2000 and in the BABAR experiment [13]. The curve is the result of the VMD fit. Right panel: The relative difference between the $e^{+} e^{-} \rightarrow K^{+} K^{-}$cross section measured in the BABAR experiment and the fit to SND data. For BABAR data (points with error bars), the statistical errors (diagonal elements of the covariance matrix) are shown. The shaded band represents the SND and BABAR systematic uncertainties combined in quad- rature.

Also some preliminary results for $e^{+} e^{-} \rightarrow \pi^{+} \pi^{-}$cross section measurement in the energy range 520-880 MeV were obtained recently. The $e^{+} e^{-} \rightarrow \pi^{+} \pi^{-}$cross section is the largest cross section below $1 \mathrm{GeV}$. Our result in comparison with most precise previous measurement by BABAR [14] is shown in Fig. 3. There is a disagreement between our results and the BABAR data above $830 \mathrm{MeV}$.

\section{Previously unmeasured cross sections}

Below $2 \mathrm{GeV}$ one can calculate the total hadronic cross section as a sum of exclusive cross sections. However, in the energy region between 1.5-2.0 GeV the exclusive data are incomplete. 


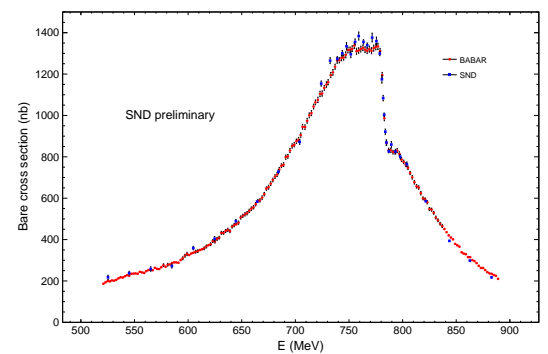

Figure 3: The $e^{+} e^{-} \rightarrow \pi^{+} \pi^{-}$cross section measured by SND at VEPP2000 (preliminary) in comparison with BABAR [14] results.

This section is devoted to some previously unstudied process.

The process $e^{+} e^{-} \rightarrow \pi^{+} \pi^{-} \pi^{0} \eta$ shows the complex internal structure. Our preliminary study reveals the presence of at least four mechanisms for this reaction: $\omega \eta, \phi \eta, a_{0}(980) \rho$, and structureless $\pi^{+} \pi^{-} \pi^{0} \eta$. The known $\omega \eta$ and $\phi \eta$ mechanisms together give about 50-60\% contribution to the cross section below $1.8 \mathrm{GeV}$. Above $a_{0} \rho$ is the dominant mechanism. Preliminary result on the $e^{+} e^{-} \rightarrow \pi^{+} \pi^{-} \pi^{0} \eta$ cross section is shown in Fig. 4 (left). The cross section for the subprocess $e^{+} e^{-} \rightarrow \omega \eta$ was measured separately [15] and it is shown in Fig. 4 (right) in comparison with the BABAR measurement [16]. Our results are more accurate and in disagreement with the BABAR data above $1.6 \mathrm{GeV}$.
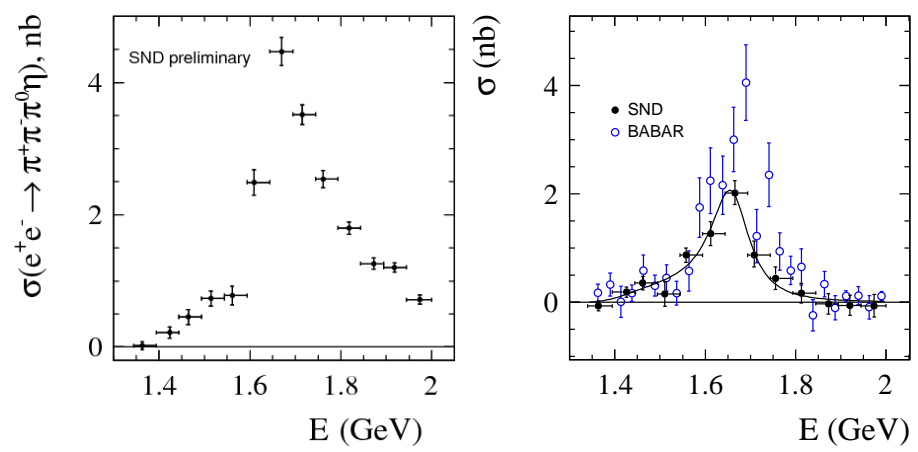

Figure 4: Left panel: The $e^{+} e^{-} \rightarrow \pi^{+} \pi^{-} \pi^{0} \eta$ cross section measured by SND (preliminary). Right panel: The $e^{+} e^{-} \rightarrow \omega \eta$ cross section measured by SND in comparison with BABAR data [16]. The curve is the result of the VMD fit.

The process $e^{+} e^{-} \rightarrow \omega \pi^{0} \eta \rightarrow \pi^{0} \pi^{0} \eta \gamma$ is studied in the seven-photon final state [17]. The analysis of $\pi^{0} \gamma$ invariant-mass distribution shows the dominance of the $\omega \pi^{0} \eta$ intermediate state. The measured $e^{+} e^{-} \rightarrow \omega \pi^{0} \eta$ cross section is shown in Fig. 5 (left). Figure 5 (right) shows that $\pi^{0} \eta$ mass distribution for selected $\omega \pi^{0} \eta$ events is well described by the simulation with $\omega a_{0}(980)$ as intermediate state.

Both processes mentioned above give a sizable contribution $(\sim 5 \%)$ to the total hadronic cross section.

\section{Acknowledgement}

This work is supported in part by the RFBR grants 16-02-00327 and 16-02-00014. Part of 

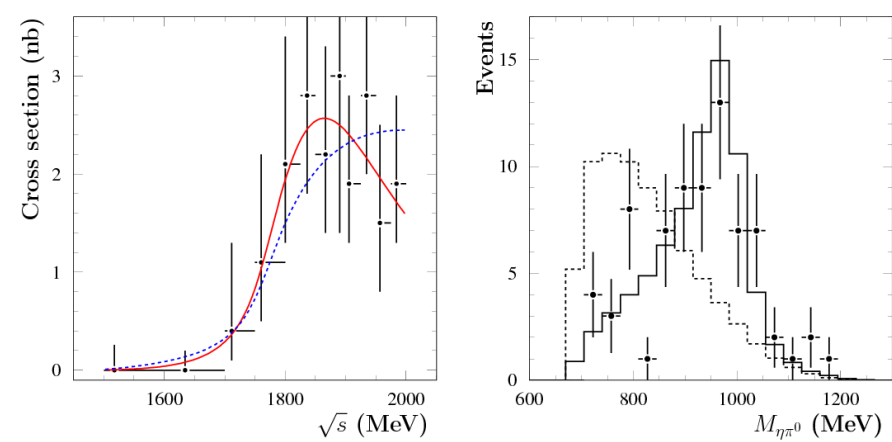

Figure 5: Left panel: The $e^{+} e^{-} \rightarrow \omega \eta \pi^{0}$ cross section measured by SND. The solid (dashed) curve shows the result of the fit in the model of $\omega a_{0}(980)$ intermediate state with (without) a resonance contribution. Right panel: The $\eta \pi^{0}$ invariant mass spectrum for selected $e^{+} e^{-} \rightarrow \omega \eta \pi^{0}$ events. The solid (dashed) histogram represents $e^{+} e^{-} \rightarrow \omega a_{0}(980)$ ( $\omega \eta \pi^{0}$ phase-space) simulation.

this work related to the photon reconstruction algorithm in the electromagnetic calorimeter is supported by the Russian Science Foundation (project No. 14-50-00080).

\section{References}

[1] M. N. Achasov et al., Nucl. Instrum. Methods Phys. Res., Sect. A 598, 31 (2009)

[2] V. M. Aulchenko et al., Nucl. Instrum. Methods Phys. Res., Sect. A 598, 102 (2009)

[3] A. Y. Barnyakov et al., JINST 9, C09023 (2014)

[4] V. M. Aulchenko et al., Nucl. Instrum. Methods Phys. Res., Sect. A 598, 340 (2009)

[5] I. A. Koop et al., in Proceedings of the Workshop on Physics and Detectors for DAPHNE, Frascati, Italy, 1999 (Frascati, 1999), p. 393

[6] A. Romanov et al., in Proceedings of Particle Accelerator Conference PAC 2013, Pasadena, CA USA, 2013 , p. 14

[7] M. N. Achasov et al. (SND Collaboration), Eur. Phys. J. C 12, 25 (2000)

[8] M. N. Achasov et al. (SND Collaboration), Phys. Lett. B 559, 171 (2003)

[9] R. R. Akhmetshin et al. (CMD-2 Collaboration), Phys. Lett. B 605, 26 (2005)

[10] M. N. Achasov et al. (SND Collaboration), Phys. Rev. D 93, 092001 (2016)

[11] F. Ambrosino et al. (KLOE Collaboration), Phys. Lett. B 669, 223 (2008)

[12] M. N. Achasov et al. (SND Collaboration), Phys. Rev. D 94, 112006 (2016)

[13] J. P. Lees et al. (BaBar Collaboration), Phys. Rev. D 88, 032013 (2013)

[14] J. P. Lees et al. (BABAR Collaboration), Phys. Rev. D 86, 032013 (2012)

[15] M. N. Achasov et al. (SND Collaboration), Phys. Rev. D 94, 092002 (2016)

[16] B. Aubert et al. (BABAR Collaboration), Phys. Rev. D 73, 052003 (2006)

[17] M. N. Achasov et al. (SND Collaboration), Phys. Rev. D 94, 032010 (2016) 